\title{
BACTERIAL FLORA OF THE JEJUNUM: A COMPARISON OF LUMINAL ASPIRATE AND MUCOSAL BIOPSY
}

\author{
P. Bhat*, M. J. Albert, D. Rajan, J. Ponniah, V. I. Mathan \\ AND S. J. BAKER $\dagger$ \\ Wellcome Research Unit $\ddagger$, Christian Medical College Hospital Vellore-632004,Tamil Nadu, India
}

IN recent years there has been an increased interest in the bacterial flora of the small intestine of man in health and disease. Studies have usually been made on aspirated luminal content, the implicit assumption being that this provides a representative sample of the whole of the bacterial population in that part of the intestine. Plaut et al. (1967) in a study of five subjects found a general similarity between the findings on culture of the biopsy and culture of the jejunal juice. However, Dubos et al. (1965) demonstrated in mice a flora intimately adherent to the gastrointestinal epithelium that did not exist free in the lumen. The present study was undertaken to compare the flora of man that could be grown from fluid aspirated from the lumen of the first part of the jejunum with the flora grown by culture of mucosal tissue obtained at biopsy from the same site.

\section{MATERIAL AND METHODS}

Forty subjects were studied; 26 patients with tropical sprue, two with a stagnant-bowel syndrome, two with malabsorption associated with agammaglobulinaemia and 10 control subjects; all gave their informed consent for the study. All the subjects belonged to the poorer socio-economic group and ate a largely vegetarian diet similar to that described by Rao and Rao (1958). They were admitted to a metabolic ward, and were given a standard vegetarian diet. As soon as possible after admission they were intubated in the fasting state with a sterile radioopaque polyvinyl tube, which was attached in parallel to a sterile Crosby capsule or a sterile Rubin's multipurpose biopsy capsule. The tip of the polyvinyl tube and biopsy capsule were positioned in the first part of the duodenum under fluoroscopic control. The Rubin's capsule was always passed with the knife in the closed position to reduce the possibility of contamination of the cutting head during its passage. Intestinal contents were aspirated from the first loop of the jejunum. The first few millilitres of the luminal contents were discarded and then the sample for study was taken. Immediately thereafter a jejunal mucosal biopsy was obtained from the same level.

Specimens of luminal contents were collected in sterile screw-capped bottles, and jejunal biopsies were transferred to small weighed pieces of sterile aluminum foil in a sterile petri dish. Both were immediately transported on ice to the laboratory. The biopsy was weighed on a

Received 9 Apr. 1979; revised version accepted 4 Sept. 1979 India.

* Present address: Department of Microbiology, St John's Medical College, Bangalore, Karnataka,

$\dagger$ Present address: Gastroenterology, University of Manitoba, St Boniface General Hospital, 409 Tache Avenue, Winnipeg, Manitoba, Canada.

$\ddagger$ Supported by the Wellcome Trust in collaboration with the World Health Organization. 
torsion balance, washed thoroughly with $2 \mathrm{ml}$ of sterile isotonic saline to reduce any surface contamination, placed in a sterile teflon tissue grinder and homogenised in $2 \mathrm{ml}$ of sterile saline. The jejunal juice, the washing fluid, and the tissue homogenate were serially diluted in six tenfold steps and cultured aerobically, anaerobically and microaerophilically on a wide variety of media as described previously (Bhat et al., 1972).

The following groups of organisms were identified by standard bacteriological procedures: enterobacteria, enterococci, other aerobic streptococci, neisseriae, staphylococci, lactobacilli, yeasts and yeast-like organisms, fusobacteria including Sphaerophorus, bacteroides including Bacteroides melaninogenicus, bifidobacteria, veillonellae and lecithinase-positive clostridia mainly Clostridium welchii. Surface viable counts were done by the spreading method (Cruickshank Duguid and Swain, 1965) with 0.1-ml inocula. Results were expressed as the logarithm to the base of 10 of the number of viable organisms per $\mathrm{ml}$ of the jejunal juice, per ml of rinsing fluid, or per $\mathrm{g}$ wet weight of tissue. No growth from the inoculum of the undiluted jejunal juice, washings or mucosal homogenate was recorded as 0 and represented less than 10 organisms $/ \mathrm{ml}$ of jejunal juice or rinsing fluid or less than 20 organisms/biopsy specimen. In our hands this technique provided a high degree of reproducibility; for replicate studies on single samples the standard deviation ranged from 0.06 to $0.20 \log _{10}$ organisms $/ \mathrm{ml}$ for the different groups of bacteria.

\section{RESULTS}

The most common groups of organisms in jejunal juice and in biopsy were enterobacteria, streptococci, veillonellae, fusobacteria and bacteroides. The types of organisms grown from the jejunal juice and the mucosal biopsy were identical in only four out of the 40 pairs of specimens (table I). In another nine pairs of specimens the flora was very similar except that one organism was

TABLE I.

Details of identical flora obtained from paired samples of jejunal juice and mucosal-biopsy homogenate from four subjects

\begin{tabular}{|c|c|c|c|c|c|c|c|c|c|c|c|c|c|}
\hline \multirow[b]{2}{*}{$\begin{array}{c}\text { Subject } \\
\text { no. }\end{array}$} & \multirow[b]{2}{*}{ Diagnosis } & \multirow[b]{2}{*}{ Specimen } & \multicolumn{11}{|c|}{$\begin{array}{l}\log _{10} \text { numbers* of organisms of the stated group } \\
\text { ml of fluid or per gram of tissue }\end{array}$} \\
\hline & & & 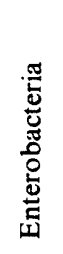 & 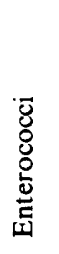 & 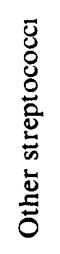 & 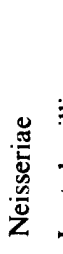 & 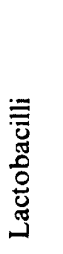 & 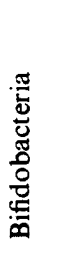 & 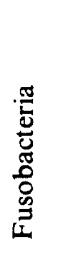 & 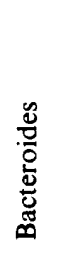 & $\begin{array}{l}\stackrel{\mathscr{\Xi}}{\bar{\Xi}} \\
\stackrel{\bar{\Xi}}{\bar{\Xi}}\end{array}$ & $\begin{array}{l}\frac{\pi}{\sigma} \\
\frac{\pi}{5} \\
\frac{0}{0}\end{array}$ & 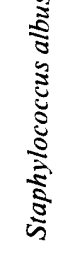 \\
\hline 1 & Sprue & $\begin{array}{l}\text { Juice } \\
\text { Biopsy }\end{array}$ & $\begin{array}{l}7 \cdot 3 \\
7 \cdot 1\end{array}$ & $\begin{array}{l}0 \\
0\end{array}$ & $\begin{array}{l}6 \cdot 8 \\
5 \cdot 8\end{array}$ & $\begin{array}{l}0 \\
0\end{array}$ & $\begin{array}{l}0 \\
0\end{array}$ & $\begin{array}{l}0 \\
0\end{array}$ & $\begin{array}{l}5 \cdot 0 \\
5 \cdot 2\end{array}$ & $\begin{array}{l}0 \\
0\end{array}$ & $\begin{array}{l}4 \cdot 9 \\
3 \cdot 3\end{array}$ & $\begin{array}{l}0 \\
0\end{array}$ & $\begin{array}{l}0 \\
0\end{array}$ \\
\hline 2 & Sprue & & $\begin{array}{l}0 \\
0\end{array}$ & $\begin{array}{l}0 \\
0\end{array}$ & $\begin{array}{l}5 \cdot 6 \\
5 \cdot 5\end{array}$ & $\begin{array}{l}0 \\
0\end{array}$ & $\begin{array}{l}0 \\
0\end{array}$ & $\begin{array}{l}0 \\
0\end{array}$ & $\begin{array}{l}4 \cdot 3 \\
3 \cdot 7\end{array}$ & $\begin{array}{l}4 \cdot 4 \\
4 \cdot 1\end{array}$ & $\begin{array}{l}3 \cdot 7 \\
3 \cdot 9\end{array}$ & $\begin{array}{l}0 \\
0\end{array}$ & $\begin{array}{l}0 \\
0\end{array}$ \\
\hline 3 & Sprue & & $\begin{array}{l}6 \cdot 6 \\
6 \cdot 7\end{array}$ & $\begin{array}{l}0 \\
0\end{array}$ & $\begin{array}{l}6 \cdot 7 \\
6 \cdot 6\end{array}$ & $\begin{array}{l}0 \\
0\end{array}$ & $\begin{array}{l}0 \\
0\end{array}$ & $\begin{array}{l}0 \\
0\end{array}$ & $\begin{array}{l}3 \cdot 8 \\
4 \cdot 0\end{array}$ & $\begin{array}{l}4 \cdot 1 \\
5 \cdot 1\end{array}$ & $\begin{array}{l}4.9 \\
4 \cdot 5\end{array}$ & $\begin{array}{l}0 \\
0\end{array}$ & $\begin{array}{l}0 \\
0\end{array}$ \\
\hline 4 & $\begin{array}{l}\text { Stagnant- } \\
\text { bowel-syndrome }\end{array}$ & $\begin{array}{l}\text { Juice } \\
\text { Biopsy }\end{array}$ & $\begin{array}{l}7.6 \\
7.6\end{array}$ & $\begin{array}{l}7 \cdot 2 \\
6 \cdot 9\end{array}$ & $\begin{array}{l}0 \\
0\end{array}$ & $\begin{array}{ll}0 & 5 \\
0 & 4\end{array}$ & $\begin{array}{l}5 \cdot 3 \\
4 \cdot 9 \\
5\end{array}$ & $\begin{array}{l}5 \cdot 3 \\
5 \cdot 7\end{array}$ & $\begin{array}{l}0 \\
0\end{array}$ & $\begin{array}{l}0 \\
0\end{array}$ & $\begin{array}{l}4.7 \\
5.7\end{array}$ & $\begin{array}{l}5 \cdot 1 \\
4 \cdot 9\end{array}$ & $\begin{array}{l}4 \cdot 7 \\
3.9\end{array}$ \\
\hline
\end{tabular}

* 0 signifies less than the minimum number detectable by the dilution procedure used (see Methods). 


\section{TABLE II}

Details of flora from paired samples of jejunal juice and mucosal biopsy homogenate from nine subjects, with one group of organisms present in one sample but absent from the other

\begin{tabular}{|c|c|c|c|c|c|c|c|c|c|c|c|c|c|c|c|}
\hline \multirow[b]{2}{*}{$\begin{array}{l}\text { Subject } \\
\text { no. }\end{array}$} & \multirow[b]{2}{*}{ Diagnosis } & \multirow[b]{2}{*}{ Specimen } & \multicolumn{13}{|c|}{$\begin{array}{c}\log _{10} \text { numbers* of organisms of the stated group per } \mathrm{ml} \text { of fluid } \\
\text { or per gram of tissue }\end{array}$} \\
\hline & & & 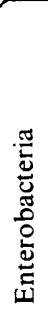 & 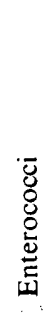 & 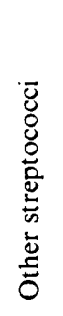 & 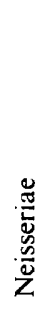 & 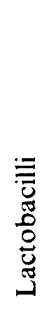 & 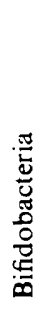 & 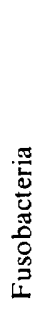 & 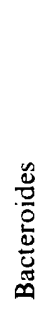 & 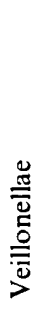 & 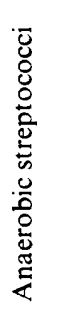 & 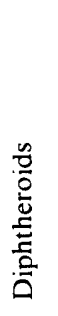 & 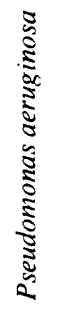 & $\begin{array}{l}\stackrel{\infty}{心} \\
\stackrel{\Xi}{\Xi}\end{array}$ \\
\hline \multirow[t]{2}{*}{5} & Sprue & Juice & $6 \cdot 7$ & 0 & $2 \cdot 3$ & 0 & 0 & $1 \cdot 3$ & 0 & $2 \cdot 7$ & 0 & 0 & 1.5 & 0 & 0 \\
\hline & & Biopsy & $4 \cdot 7$ & 0 & $2 \cdot 9$ & 0 & 0 & $3 \cdot 2$ & 0 & $2 \cdot 9$ & 0 & 0 & 0 & 0 & 0 \\
\hline \multirow[t]{2}{*}{6} & Sprue & Juice & $2 \cdot 0$ & 0 & $4 \cdot 1$ & $3 \cdot 3$ & 0 & 0 & $2 \cdot 0$ & 0 & 3.0 & 0 & 0 & $2 \cdot 0$ & 0 \\
\hline & & Biopsy & $4 \cdot 1$ & 0 & $5 \cdot 9$ & $5 \cdot 9$ & 0 & 0 & $3 \cdot 6$ & 0 & 0 & 0 & 0 & $3 \cdot 7$ & 0 \\
\hline \multirow[t]{2}{*}{7} & Sprue & Juice & 0 & 0 & $4 \cdot 5$ & $4 \cdot 8$ & $1 \cdot 0$ & $2 \cdot 5$ & $3 \cdot 7$ & $4 \cdot 0$ & $3 \cdot 8$ & 0 & $3 \cdot 3$ & 0 & $1 \cdot 0$ \\
\hline & & Biopsy & 0 & 0 & $6 \cdot 2$ & $6 \cdot 2$ & 6.8 & $5 \cdot 0$ & $5 \cdot 8$ & 0 & $6 \cdot 3$ & 0 & $5 \cdot 7$ & 0 & $4 \cdot 1$ \\
\hline \multirow[t]{2}{*}{8} & Stagnant- & Juice & $3 \cdot 3$ & 0 & $5 \cdot 1$ & 0 & $4 \cdot 4$ & $4 \cdot 7$ & 0 & 0 & $5 \cdot 0$ & 0 & 0 & 0 & 0 \\
\hline & $\begin{array}{l}\text { loop } \\
\text { syndrome }\end{array}$ & Biopsy & 0 & 0 & $3 \cdot 7$ & 0 & $3 \cdot 7$ & $4 \cdot 2$ & 0 & 0 & 3.9 & 0 & 0 & 0 & 0 \\
\hline \multirow[t]{2}{*}{9} & Sprue & Juice & $3 \cdot 3$ & 0 & $3 \cdot 3$ & $3 \cdot 4$ & 0 & 0 & $2 \cdot 3$ & 0 & $2 \cdot 8$ & 0 & 0 & 0 & 0 \\
\hline & & Biopsy & $4 \cdot 3$ & 0 & $5 \cdot 7$ & $4 \cdot 8$ & 0 & 0 & $3 \cdot 3$ & 3.0 & $3 \cdot 3$ & 0 & 0 & 0 & 0 \\
\hline \multirow[t]{2}{*}{10} & Sprue & Juice & $6 \cdot 8$ & $4 \cdot 8$ & 0 & 0 & $4 \cdot 5$ & $4 \cdot 1$ & $3 \cdot 3$ & 0 & $4 \cdot 2$ & 0 & 0 & $6 \cdot 7$ & 0 \\
\hline & & Biopsy & $7 \cdot 4$ & $5 \cdot 3$ & 0 & 0 & $3 \cdot 9$ & $3 \cdot 9$ & $3 \cdot 4$ & 3.5 & $4 \cdot 6$ & 0 & 0 & $7 \cdot 0$ & 0 \\
\hline \multirow[t]{2}{*}{11} & Sprue & Juice & $2 \cdot 0$ & 0 & $2 \cdot 0$ & $2 \cdot 3$ & $2 \cdot 0$ & 0 & 0 & 0 & 0 & 0 & 0 & 0 & 0 \\
\hline & & Biopsy & $3 \cdot 5$ & 0 & $5 \cdot 6$ & 4.0 & $3 \cdot 4$ & 0 & 0 & 0 & 0 & 0 & 0 & 0 & 3.4 \\
\hline \multirow[t]{2}{*}{12} & Hypogamma- & Juice & 6.9 & 0 & $6 \cdot 8$ & 0 & $3 \cdot 3$ & $2 \cdot 9$ & $5 \cdot 1$ & 4.9 & $\mathbf{0}$ & $6 \cdot 4$ & 0 & 0 & 0 \\
\hline & $\begin{array}{l}\text { globulin- } \\
\text { aemia }\end{array}$ & Biopsy & $5 \cdot 8$ & 0 & $6 \cdot 2$ & $6 \cdot 6$ & 0 & 0 & $3 \cdot 8$ & 0 & 4.9 & 0 & 0 & 0 & 0 \\
\hline \multirow[t]{2}{*}{13} & Agamma- & Juice & $5 \cdot 1$ & 0 & $4 \cdot 3$ & 0 & 0 & 0 & $2 \cdot 3$ & 0 & $4 \cdot 4$ & 0 & 0 & 0 & 0 \\
\hline & $\begin{array}{l}\text { globulin- } \\
\text { aemia }\end{array}$ & Biopsy & $5 \cdot 8$ & 0 & $6 \cdot 2$ & $6 \cdot 6$ & 0 & 0 & $3 \cdot 8$ & 0 & $4 \cdot 9$ & 0 & 0 & 0 & 0 \\
\hline
\end{tabular}

* The 'discrepant' results are shown in bold type; 0 signifies less than the minimum number detectable by the dilution procedure used (see Methods). 


\section{TABLE III}

Details of flora from paired samples of jejunal juice and mucosal-biopsy homogenate from six subjects, with two groups of organisms present in one sample but absent from the other

\begin{tabular}{|c|c|c|c|c|c|c|c|c|c|c|c|c|c|c|c|c|}
\hline \multirow[b]{2}{*}{$\begin{array}{c}\text { Subject } \\
\text { no. }\end{array}$} & \multirow[b]{2}{*}{ Diagnosis } & \multirow[b]{2}{*}{ Specimen } & \multicolumn{14}{|c|}{$\begin{array}{c}\log _{10} \text { numbers* of organisms of the stated group } \\
\text { per ml of fluid or per gram of tissue }\end{array}$} \\
\hline & & & 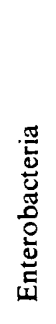 & 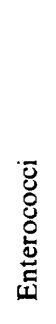 & 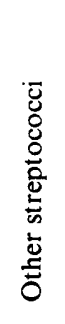 & 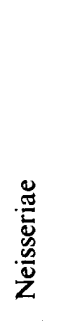 & 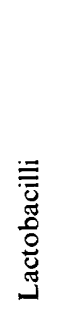 & 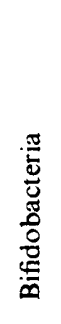 & 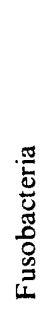 & 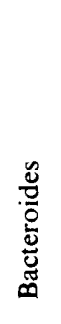 & 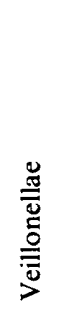 & 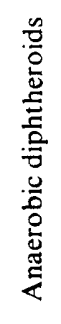 & 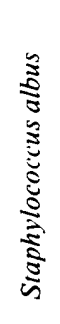 & 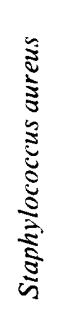 & 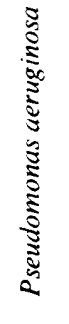 & 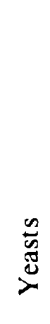 \\
\hline 14 & Sprue & $\begin{array}{l}\text { Juice } \\
\text { Biopsy }\end{array}$ & $\begin{array}{l}5 \cdot 6 \\
6 \cdot 0\end{array}$ & $\begin{array}{l}4 \cdot 9 \\
5 \cdot 3\end{array}$ & $\begin{array}{l}5 \cdot 5 \\
6 \cdot 1\end{array}$ & $\begin{array}{l}0 \\
0\end{array}$ & $\begin{array}{l}0 \\
0\end{array}$ & $\begin{array}{l}5 \cdot 2 \\
4 \cdot 9\end{array}$ & $\begin{array}{l}2 \cdot 3 \\
4 \cdot 5\end{array}$ & $\begin{array}{c}4.6 \\
0\end{array}$ & $\begin{array}{l}4 \cdot 0 \\
6 \cdot 1\end{array}$ & $\begin{array}{l}0 \\
0\end{array}$ & $\begin{array}{l}0 \\
0\end{array}$ & $\begin{array}{l}0 \\
0\end{array}$ & $\begin{array}{c}0 \\
5 \cdot 2\end{array}$ & $\begin{array}{l}0 \\
0\end{array}$ \\
\hline 15 & Sprue & $\begin{array}{l}\text { Juice } \\
\text { Biopsy }\end{array}$ & $\begin{array}{l}5 \cdot 6 \\
6 \cdot 1\end{array}$ & $\begin{array}{l}0 \\
0\end{array}$ & $\begin{array}{l}5 \cdot 6 \\
5 \cdot 6\end{array}$ & $\begin{array}{c}5 \cdot 6 \\
0\end{array}$ & $\begin{array}{l}0 \\
0\end{array}$ & $\begin{array}{l}2 \cdot 5 \\
4 \cdot 2\end{array}$ & $\begin{array}{l}4 \cdot 8 \\
4 \cdot 9\end{array}$ & $\begin{array}{l}0 \\
0\end{array}$ & $\begin{array}{l}4 \cdot 8 \\
4 \cdot 8\end{array}$ & $\begin{array}{l}0 \\
0\end{array}$ & $\begin{array}{l}0 \\
0\end{array}$ & $\begin{array}{l}0 \\
0\end{array}$ & $\begin{array}{c}5.4 \\
0\end{array}$ & $\begin{array}{l}0 \\
0\end{array}$ \\
\hline 16 & Sprue & $\begin{array}{l}\text { Juice } \\
\text { Biopsy }\end{array}$ & $\begin{array}{l}3.4 \\
3 \cdot 7\end{array}$ & $\begin{array}{l}0 \\
0\end{array}$ & $\begin{array}{l}5.0 \\
5.9\end{array}$ & $\begin{array}{c}0 \\
6.0\end{array}$ & $\begin{array}{l}0 \\
0\end{array}$ & $\begin{array}{l}0 \\
0\end{array}$ & $\begin{array}{l}4 \cdot 7 \\
3 \cdot 2\end{array}$ & $\begin{array}{l}5 \cdot 6 \\
4.9\end{array}$ & $\begin{array}{l}4 \cdot 2 \\
3 \cdot 0\end{array}$ & $\begin{array}{l}0 \\
0\end{array}$ & $\begin{array}{l}0 \\
0\end{array}$ & $\begin{array}{c}0 \\
5 \cdot 5\end{array}$ & $\begin{array}{l}0 \\
0\end{array}$ & $\begin{array}{l}0 \\
0\end{array}$ \\
\hline 17 & Sprue & $\begin{array}{l}\text { Juice } \\
\text { Biopsy }\end{array}$ & $\begin{array}{l}0 \\
0\end{array}$ & $\begin{array}{l}0 \\
0\end{array}$ & $\begin{array}{l}2 \cdot 1 \\
4 \cdot 4\end{array}$ & $\begin{array}{c}0 \\
4 \cdot 9\end{array}$ & $\begin{array}{l}0 \\
0\end{array}$ & $\begin{array}{l}0 \\
0\end{array}$ & $\begin{array}{l}0 \\
0\end{array}$ & $\begin{array}{l}0 \\
0\end{array}$ & $\begin{array}{l}2 \cdot 0 \\
4 \cdot 5\end{array}$ & $\begin{array}{c}0 \\
3.0\end{array}$ & $\begin{array}{l}1 \cdot 3 \\
1 \cdot 3\end{array}$ & $\begin{array}{l}0 \\
0\end{array}$ & $\begin{array}{l}0 \\
0\end{array}$ & $\begin{array}{l}2 \cdot 3 \\
3 \cdot 0\end{array}$ \\
\hline 18 & Sprue & $\begin{array}{l}\text { Juice } \\
\text { Biopsy }\end{array}$ & $\begin{array}{l}4.5 \\
6.8\end{array}$ & $\begin{array}{l}3 \cdot 3 \\
5 \cdot 4\end{array}$ & $\begin{array}{l}4 \cdot 1 \\
5 \cdot 9\end{array}$ & $\begin{array}{c}0 \\
6 \cdot 7\end{array}$ & $\begin{array}{c}0 \\
3 \cdot 4\end{array}$ & $\begin{array}{l}0 \\
0\end{array}$ & $\begin{array}{l}2 \cdot 9 \\
3 \cdot 1\end{array}$ & $\begin{array}{l}0 \\
0\end{array}$ & $\begin{array}{l}2 \cdot 0 \\
3 \cdot 5\end{array}$ & $\begin{array}{l}0 \\
0\end{array}$ & $\begin{array}{l}0 \\
0\end{array}$ & $\begin{array}{l}0 \\
0\end{array}$ & $\begin{array}{l}0 \\
0\end{array}$ & $\begin{array}{l}0 \\
0\end{array}$ \\
\hline 19 & Control & $\begin{array}{l}\text { Juice } \\
\text { Biopsy }\end{array}$ & $\begin{array}{l}2 \cdot 5 \\
5 \cdot 0\end{array}$ & $\begin{array}{c}0 \\
3 \cdot 7\end{array}$ & $\begin{array}{l}6.9 \\
7.0\end{array}$ & $\begin{array}{l}6 \cdot 7 \\
6 \cdot 3\end{array}$ & $\begin{array}{l}4 \cdot 3 \\
3 \cdot 8\end{array}$ & $\begin{array}{l}5 \cdot 6 \\
4 \cdot 0\end{array}$ & $\begin{array}{l}5 \cdot 3 \\
6 \cdot 3\end{array}$ & $\begin{array}{l}8 \cdot 3 \\
6 \cdot 4\end{array}$ & $\begin{array}{l}6 \cdot 3 \\
5 \cdot 5\end{array}$ & $\begin{array}{l}0 \\
0\end{array}$ & $\begin{array}{l}0 \\
0\end{array}$ & $\begin{array}{l}0 \\
0\end{array}$ & $\begin{array}{c}5 \cdot 1 \\
0\end{array}$ & $\begin{array}{l}0 \\
0\end{array}$ \\
\hline
\end{tabular}

* See footnotes to table II. 
TABLE IV

Details of flora from paired samples of jejunal juice and mucosal-biopsy homogenate from 11 subjects, with three or more groups of organisms present in one sample but absent from the other

\begin{tabular}{|c|c|c|c|c|c|c|c|c|c|c|c|c|c|c|c|c|}
\hline \multirow[b]{2}{*}{$\begin{array}{l}\text { Subject } \\
\text { no. }\end{array}$} & \multirow[b]{2}{*}{ Diagnosis } & \multirow[b]{2}{*}{ Specimen } & \multicolumn{14}{|c|}{$\begin{array}{l}\log _{10} \text { numbers* of organisms of the stated group } \\
\text { per ml of fluid or per gram of tissue }\end{array}$} \\
\hline & & & 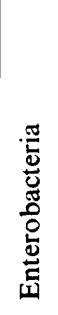 & 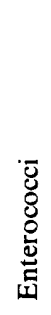 & 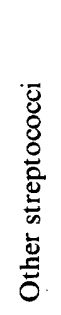 & 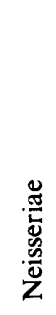 & 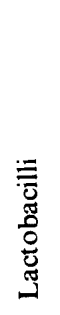 & 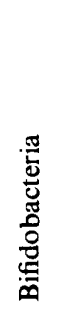 & 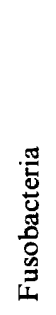 & 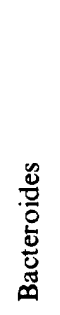 & 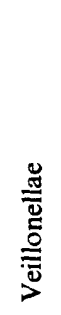 & 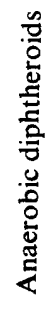 & 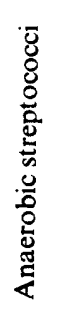 & 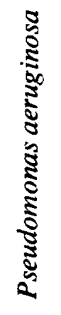 & 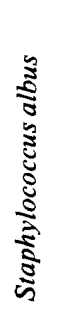 & 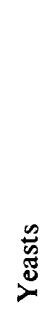 \\
\hline 20 & Sprue & $\begin{array}{l}\text { Juice } \\
\text { Biopsy }\end{array}$ & $\begin{array}{l}2 \cdot 9 \\
5 \cdot 1\end{array}$ & $\begin{array}{l}0 \\
0\end{array}$ & $\begin{array}{l}4 \cdot 5 \\
5 \cdot 9\end{array}$ & $\begin{array}{c}0 \\
4 \cdot 3\end{array}$ & $\begin{array}{l}0 \\
0\end{array}$ & $\begin{array}{l}0 \\
0\end{array}$ & $\begin{array}{l}3 \cdot 9 \\
3 \cdot 6\end{array}$ & $\begin{array}{l}0 \\
0\end{array}$ & $\begin{array}{l}5 \cdot 0 \\
3 \cdot 0\end{array}$ & $\begin{array}{c}3.7 \\
0\end{array}$ & $\begin{array}{l}0 \\
0\end{array}$ & $\begin{array}{l}0 \\
0\end{array}$ & $\begin{array}{c}3 \cdot 3 \\
0\end{array}$ & $2 \cdot 3$ \\
\hline 21 & Sprue & $\begin{array}{l}\text { Juice } \\
\text { Biopsy }\end{array}$ & $\begin{array}{l}6 \cdot 2 \\
5 \cdot 8\end{array}$ & $2 \cdot 5$ & $\begin{array}{l}5 \cdot 5 \\
5 \cdot 7\end{array}$ & $\begin{array}{l}0 \\
0\end{array}$ & $\begin{array}{c}2.8 \\
0\end{array}$ & $\begin{array}{c}0 \\
4 \cdot 6\end{array}$ & $\begin{array}{l}0 \\
0\end{array}$ & $\begin{array}{l}6 \cdot 0 \\
5 \cdot 5\end{array}$ & $\begin{array}{l}0 \\
0\end{array}$ & $\begin{array}{l}0 \\
0\end{array}$ & $\begin{array}{l}5 \cdot 5 \\
5 \cdot 6\end{array}$ & $\begin{array}{c}4.8 \\
0\end{array}$ & $\begin{array}{l}0 \\
0\end{array}$ & $\begin{array}{l}0 \\
0\end{array}$ \\
\hline 22 & Sprue & $\begin{array}{l}\text { Juice } \\
\text { Biopsy }\end{array}$ & $\begin{array}{l}0 \\
0\end{array}$ & $\begin{array}{l}0 \\
0\end{array}$ & $\begin{array}{l}6 \cdot 4 \\
5 \cdot 2\end{array}$ & $\begin{array}{c}0 \\
4 \cdot 8\end{array}$ & $\begin{array}{l}0 \\
0\end{array}$ & $\begin{array}{l}0 \\
0\end{array}$ & $\begin{array}{c}4 \cdot 5 \\
0\end{array}$ & $\begin{array}{l}5 \cdot 3 \\
3 \cdot 5\end{array}$ & $\begin{array}{l}5 \cdot 1 \\
5 \cdot 0\end{array}$ & $\begin{array}{c}0 \\
3 \cdot 5\end{array}$ & $\begin{array}{l}0 \\
0\end{array}$ & $\begin{array}{l}0 \\
0\end{array}$ & $\begin{array}{l}0 \\
0\end{array}$ & $\begin{array}{l}0 \\
0\end{array}$ \\
\hline 23 & Control & $\begin{array}{l}\text { Juice } \\
\text { Biopsy }\end{array}$ & $\begin{array}{l}4 \cdot 7 \\
5 \cdot 6\end{array}$ & $\begin{array}{l}3 \cdot 7 \\
5 \cdot 1\end{array}$ & $\begin{array}{l}0 \\
0\end{array}$ & $\begin{array}{l}0 \\
0\end{array}$ & $\begin{array}{l}0 \\
0\end{array}$ & $\begin{array}{c}0 \\
4 \cdot 1\end{array}$ & $\begin{array}{c}0 \\
3 \cdot 4\end{array}$ & $\begin{array}{l}0 \\
0\end{array}$ & $\begin{array}{c}0 \\
4 \cdot 2\end{array}$ & $\begin{array}{l}0 \\
0\end{array}$ & $\begin{array}{l}0 \\
0\end{array}$ & $\begin{array}{l}0 \\
0\end{array}$ & $\begin{array}{l}0 \\
0\end{array}$ & $\begin{array}{l}0 \\
0\end{array}$ \\
\hline 24 & Control & $\begin{array}{l}\text { Juice } \\
\text { Biopsy }\end{array}$ & $\begin{array}{c}2.9 \\
0\end{array}$ & $\begin{array}{l}0 \\
0\end{array}$ & $\begin{array}{l}2 \cdot 6 \\
5 \cdot 8\end{array}$ & $\begin{array}{c}0 \\
4 \cdot 2\end{array}$ & $\begin{array}{c}3.0 \\
0\end{array}$ & $\begin{array}{c}2 \cdot 5 \\
0\end{array}$ & $\begin{array}{c}0 \\
4 \cdot 4\end{array}$ & $\begin{array}{l}1 \cdot 6 \\
4 \cdot 4\end{array}$ & $\begin{array}{c}0 \\
4.9\end{array}$ & $\begin{array}{l}0 \\
0\end{array}$ & $\begin{array}{c}2.8 \\
0\end{array}$ & $\begin{array}{l}0 \\
0\end{array}$ & $\begin{array}{l}0 \\
0\end{array}$ & $\begin{array}{l}0 \\
0\end{array}$ \\
\hline 25 & Control & $\begin{array}{l}\text { Juice } \\
\text { Biopsy }\end{array}$ & $\begin{array}{c}2 \cdot 2 \\
0\end{array}$ & $\begin{array}{l}0 \\
0\end{array}$ & $\begin{array}{l}3 \cdot 6 \\
4 \cdot 5\end{array}$ & $\begin{array}{l}2 \cdot 7 \\
4 \cdot 6\end{array}$ & $\begin{array}{l}0 \\
0\end{array}$ & $\begin{array}{c}0 \\
5.9\end{array}$ & $\begin{array}{l}1 \cdot 0 \\
4 \cdot 1\end{array}$ & $\begin{array}{l}1.3 \\
5.0\end{array}$ & $\begin{array}{c}0 \\
3 \cdot 8\end{array}$ & $\begin{array}{l}0 \\
0\end{array}$ & $\begin{array}{l}2.6 \\
3.9\end{array}$ & $\begin{array}{l}0 \\
0\end{array}$ & $\begin{array}{l}2 \cdot 2 \\
4 \cdot 2\end{array}$ & $\begin{array}{l}0 \\
0\end{array}$ \\
\hline 26 & Control & $\begin{array}{l}\text { Juice } \\
\text { Biopsy }\end{array}$ & $\begin{array}{l}0 \\
0\end{array}$ & $\begin{array}{l}0 \\
0\end{array}$ & $\begin{array}{l}4.9 \\
6 \cdot 0\end{array}$ & $\begin{array}{c}0 \\
6 \cdot 2\end{array}$ & $\begin{array}{l}0 \\
0\end{array}$ & $\begin{array}{c}1.5 \\
0\end{array}$ & $\begin{array}{c}2.5 \\
0\end{array}$ & $\begin{array}{c}0 \\
4 \cdot 6\end{array}$ & $\begin{array}{l}0 \\
0\end{array}$ & $\begin{array}{l}0 \\
0\end{array}$ & $\begin{array}{c}0 \\
5 \cdot 5\end{array}$ & $\stackrel{2 \cdot 3}{0}$ & $\begin{array}{l}0 \\
0\end{array}$ & $\begin{array}{l}0 \\
0\end{array}$ \\
\hline 27 & Control & $\begin{array}{l}\text { Juice } \\
\text { Biopsy }\end{array}$ & $\begin{array}{l}0 \\
0\end{array}$ & $\begin{array}{l}0 \\
0\end{array}$ & $\begin{array}{l}1 \cdot 8 \\
4 \cdot 4\end{array}$ & $\begin{array}{c}0 \\
3 \cdot 8\end{array}$ & $\begin{array}{l}2 \cdot 1 \\
4 \cdot 1\end{array}$ & $\begin{array}{l}1 \cdot 3 \\
4 \cdot 5\end{array}$ & $\begin{array}{c}2 \cdot 0 \\
0\end{array}$ & $\begin{array}{l}1 \cdot 6 \\
4 \cdot 9\end{array}$ & $\begin{array}{l}2 \cdot 4 \\
4 \cdot 7\end{array}$ & $\begin{array}{l}0 \\
0\end{array}$ & $\begin{array}{c}0 \\
4 \cdot 3\end{array}$ & $\begin{array}{l}0 \\
0\end{array}$ & $\begin{array}{c}0 \\
3 \cdot 6\end{array}$ & $\begin{array}{l}0 \\
0\end{array}$ \\
\hline 28 & Control & $\begin{array}{l}\text { Juice } \\
\text { Biopsy }\end{array}$ & $\begin{array}{l}0 \\
0\end{array}$ & $\begin{array}{l}0 \\
0\end{array}$ & $\begin{array}{l}2.4 \\
5.9\end{array}$ & $\begin{array}{l}3 \cdot 2 \\
5 \cdot 3\end{array}$ & $\begin{array}{l}0 \\
0\end{array}$ & $\begin{array}{c}0 \\
3 \cdot 5\end{array}$ & $\begin{array}{c}0 \\
3 \cdot 6\end{array}$ & $\begin{array}{c}0 \\
3.9\end{array}$ & $\begin{array}{c}0 \\
4 \cdot 2\end{array}$ & $\begin{array}{l}0 \\
0\end{array}$ & $\begin{array}{l}0 \\
0\end{array}$ & $\begin{array}{l}0 \\
0\end{array}$ & $\begin{array}{l}0 \\
0\end{array}$ & $\begin{array}{l}0 \\
0\end{array}$ \\
\hline 29 & Control & $\begin{array}{l}\text { Juice } \\
\text { Biopsy }\end{array}$ & $\begin{array}{c}0 \\
3.8\end{array}$ & $\begin{array}{l}0 \\
0\end{array}$ & $\begin{array}{l}3 \cdot 1 \\
3 \cdot 6\end{array}$ & $\begin{array}{l}2 \cdot 7 \\
2 \cdot 6\end{array}$ & $\begin{array}{l}3 \cdot 3 \\
3 \cdot 8\end{array}$ & $\begin{array}{l}2 \cdot 7 \\
3 \cdot 8\end{array}$ & $\begin{array}{l}0 \\
0\end{array}$ & $\begin{array}{c}1.5 \\
0\end{array}$ & $\begin{array}{l}1.0 \\
3.5\end{array}$ & $\begin{array}{l}0 \\
0\end{array}$ & $\begin{array}{l}0 \\
0\end{array}$ & $\begin{array}{c}0 \\
4 \cdot 6\end{array}$ & $\begin{array}{l}0 \\
0\end{array}$ & $\begin{array}{l}1 \cdot 7 \\
3 \cdot 3\end{array}$ \\
\hline 30 & Sprue & $\begin{array}{l}\text { Juice } \\
\text { Biopsy }\end{array}$ & $\begin{array}{l}4 \cdot 5 \\
4 \cdot 8\end{array}$ & $\begin{array}{l}0 \\
0\end{array}$ & $\begin{array}{c}0 \\
5.6\end{array}$ & $\begin{array}{l}0 \\
0\end{array}$ & $\begin{array}{l}0 \\
0\end{array}$ & $\begin{array}{l}0 \\
0\end{array}$ & $\begin{array}{c}0 \\
3 \cdot 5\end{array}$ & $\begin{array}{c}0 \\
3.7\end{array}$ & $\begin{array}{c}0 \\
3 \cdot 1\end{array}$ & $\begin{array}{l}0 \\
0\end{array}$ & $\begin{array}{c}0 \\
5.9\end{array}$ & $\begin{array}{l}0 \\
0\end{array}$ & $\begin{array}{l}0 \\
0\end{array}$ & $\begin{array}{l}0 \\
0\end{array}$ \\
\hline
\end{tabular}

* See footnotes to table II. 


\section{TABLE V}

Details of flora from related samples of jejunal juice, mucosal washings and mucosal biopsy homogenate from nine subjects, with one sample yielding no growth* or only one group of organisms despite significant yields from one or both of the other samples

\begin{tabular}{|c|c|c|c|c|c|c|c|c|c|c|c|c|c|c|c|c|}
\hline \multirow[b]{2}{*}{$\begin{array}{c}\text { Subject } \\
\text { no. }\end{array}$} & \multirow[b]{2}{*}{$\begin{array}{l}\text { Diag- } \\
\text { nosis }\end{array}$} & \multirow[b]{2}{*}{ Specimen } & \multicolumn{14}{|c|}{$\begin{array}{l}\log _{10} \text { numbers* of organisms of the stated group } \\
\text { per ml of tissue or per } g \text { of tissue }\end{array}$} \\
\hline & & & 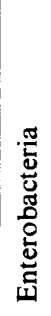 & 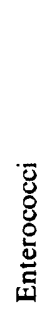 & 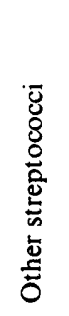 & 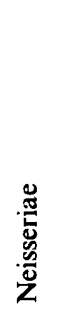 & 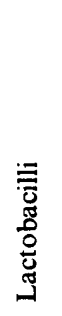 & 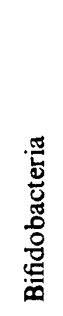 & 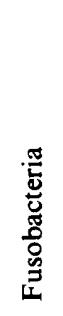 & 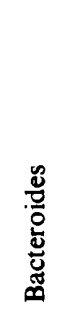 & 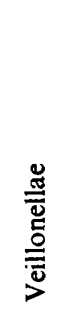 & 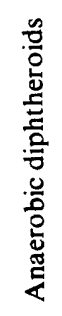 & 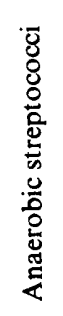 & 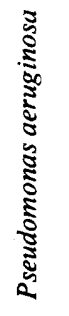 & 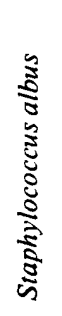 & $\stackrel{\mathscr{n}}{\ddot{d}}$ \\
\hline 31 & Sprue & $\begin{array}{l}\text { Juice } \\
\text { Biopsy } \\
\text { Saline wash }\end{array}$ & $\begin{array}{c}3 \cdot 5 \\
0 \\
0\end{array}$ & $\begin{array}{l}0 \\
0 \\
0\end{array}$ & $\begin{array}{c}0 \\
5 \cdot 1 \\
0\end{array}$ & $\begin{array}{c}0 \\
5 \cdot 3 \\
0\end{array}$ & $\begin{array}{l}0 \\
0 \\
0\end{array}$ & $\begin{array}{l}0 \\
0 \\
0\end{array}$ & $\begin{array}{c}0 \\
3 \cdot 2 \\
0\end{array}$ & $\begin{array}{c}0 \\
4 \cdot 2 \\
0\end{array}$ & $\begin{array}{c}0 \\
4 \cdot 0 \\
0\end{array}$ & $\begin{array}{l}0 \\
0 \\
0\end{array}$ & $\begin{array}{l}0 \\
0 \\
0\end{array}$ & $\begin{array}{l}0 \\
0 \\
0\end{array}$ & $\begin{array}{l}0 \\
0 \\
0\end{array}$ & $\begin{array}{c}0 \\
4.0 \\
0\end{array}$ \\
\hline 32 & Sprue & $\begin{array}{l}\text { Juice } \\
\text { Biopsy } \\
\text { Saline wash }\end{array}$ & $\begin{array}{l}0 \\
0 \\
0\end{array}$ & $\begin{array}{l}0 \\
0 \\
0\end{array}$ & $\begin{array}{c}0 \\
4 \cdot 2 \\
1.0\end{array}$ & $\begin{array}{c}0 \\
3 \cdot 9 \\
0\end{array}$ & $\begin{array}{c}0 \\
3 \cdot 6 \\
0\end{array}$ & $\begin{array}{l}0 \\
0 \\
0\end{array}$ & $\begin{array}{l}0 \\
0 \\
0\end{array}$ & $\begin{array}{c}0 \\
3 \cdot 5 \\
0\end{array}$ & $\begin{array}{c}0 \\
3 \cdot 3 \\
0\end{array}$ & $\begin{array}{c}0 \\
3.5 \\
1.0\end{array}$ & $\begin{array}{c}0 \\
4 \cdot 3 \\
0\end{array}$ & $\begin{array}{c}4 \cdot 3 \\
0 \\
0\end{array}$ & $\begin{array}{c}0 \\
3 \cdot 7 \\
0\end{array}$ & $\begin{array}{l}0 \\
0 \\
0\end{array}$ \\
\hline 33 & Sprue & $\begin{array}{l}\text { Juice } \\
\text { Biopsy } \\
\text { Saline wash }\end{array}$ & $\begin{array}{l}0 \\
0 \\
0\end{array}$ & $\begin{array}{l}0 \\
0 \\
0\end{array}$ & $\begin{array}{c}0 \\
5 \cdot 1 \\
2 \cdot 6\end{array}$ & $\begin{array}{c}0 \\
4 \cdot 3 \\
1 \cdot 8\end{array}$ & $\begin{array}{l}0 \\
0 \\
0\end{array}$ & $\begin{array}{c}0 \\
3.0 \\
0\end{array}$ & $\begin{array}{c}0 \\
3 \cdot 3 \\
0\end{array}$ & $\begin{array}{c}0 \\
3 \cdot 3 \\
0\end{array}$ & $\begin{array}{c}0 \\
2 \cdot 7 \\
0\end{array}$ & $\begin{array}{l}0 \\
0 \\
0\end{array}$ & $\begin{array}{l}0 \\
0 \\
0\end{array}$ & $\begin{array}{l}0 \\
0 \\
0\end{array}$ & $\begin{array}{l}0 \\
0 \\
0\end{array}$ & $\begin{array}{l}0 \\
0 \\
0\end{array}$ \\
\hline 34 & Sprue & $\begin{array}{l}\text { Juice } \\
\text { Biopsy } \\
\text { Saline wash }\end{array}$ & $\begin{array}{l}0 \\
0 \\
0\end{array}$ & $\begin{array}{l}0 \\
0 \\
0\end{array}$ & $\begin{array}{c}0 \\
4.8 \\
1.0\end{array}$ & $\begin{array}{c}0 \\
0 \\
2 \cdot 0\end{array}$ & $\begin{array}{l}0 \\
0 \\
0\end{array}$ & $\begin{array}{l}0 \\
0 \\
0\end{array}$ & $\begin{array}{c}0 \\
4 \cdot 1 \\
0\end{array}$ & $\begin{array}{c}0 \\
3 \cdot 8 \\
0\end{array}$ & $\begin{array}{l}0 \\
0 \\
0\end{array}$ & $\begin{array}{c}0 \\
2 \cdot 8 \\
0\end{array}$ & $\begin{array}{l}0 \\
0 \\
0\end{array}$ & $\begin{array}{l}0 \\
0 \\
0\end{array}$ & $\begin{array}{l}0 \\
0 \\
0\end{array}$ & $\begin{array}{l}0 \\
0 \\
0\end{array}$ \\
\hline 35 & Sprue & $\begin{array}{l}\text { Juice } \\
\text { Biopsy } \\
\text { Saline wash }\end{array}$ & $\begin{array}{l}0 \\
0 \\
0\end{array}$ & $\begin{array}{l}0 \\
0 \\
0\end{array}$ & $\begin{array}{c}0 \\
4 \cdot 6 \\
1.0\end{array}$ & $\begin{array}{c}0 \\
0 \\
2 \cdot 0\end{array}$ & $\begin{array}{c}0 \\
3 \cdot 7 \\
0\end{array}$ & $\begin{array}{c}0 \\
4 \cdot 0 \\
0\end{array}$ & $\begin{array}{c}0 \\
4 \cdot 0 \\
0\end{array}$ & $\begin{array}{c}0 \\
4 \cdot 0 \\
0\end{array}$ & $\begin{array}{c}0 \\
3.9 \\
0\end{array}$ & $\begin{array}{l}0 \\
0 \\
0\end{array}$ & $\begin{array}{l}0 \\
0 \\
0\end{array}$ & $\begin{array}{l}0 \\
0 \\
0\end{array}$ & $\begin{array}{l}0 \\
0 \\
0\end{array}$ & $\begin{array}{l}0 \\
0 \\
0\end{array}$ \\
\hline 36 & Sprue & $\begin{array}{l}\text { Juice } \\
\text { Biopsy } \\
\text { Saline wash }\end{array}$ & $\begin{array}{l}0 \\
0 \\
0\end{array}$ & $\begin{array}{l}0 \\
0 \\
0\end{array}$ & $\begin{array}{c}0 \\
5 \cdot 3 \\
2 \cdot 9\end{array}$ & $\begin{array}{l}0 \\
0 \\
0\end{array}$ & $\begin{array}{l}0 \\
0 \\
0\end{array}$ & $\begin{array}{l}0 \\
0 \\
0\end{array}$ & $\begin{array}{c}0 \\
4 \cdot 2 \\
1 \cdot 3\end{array}$ & $\begin{array}{c}0 \\
4 \cdot 1 \\
1 \cdot 0\end{array}$ & $\begin{array}{c}0 \\
4.6 \\
1.0\end{array}$ & $\begin{array}{l}0 \\
0 \\
0\end{array}$ & $\begin{array}{l}0 \\
0 \\
0\end{array}$ & $\begin{array}{c}0 \\
2 \cdot 7 \\
0\end{array}$ & $\begin{array}{l}0 \\
0 \\
0\end{array}$ & $\begin{array}{l}0 \\
0 \\
0\end{array}$ \\
\hline 37 & Sprue & $\begin{array}{l}\text { Juice } \\
\text { Biopsy } \\
\text { Saline wash }\end{array}$ & $\begin{array}{c}0 \\
4 \cdot 1 \\
0\end{array}$ & $\begin{array}{l}0 \\
0 \\
0\end{array}$ & $\begin{array}{l}0 \\
0 \\
0\end{array}$ & $\begin{array}{l}0 \\
0 \\
0\end{array}$ & $\begin{array}{l}0 \\
0 \\
0\end{array}$ & $\begin{array}{l}0 \\
0 \\
0\end{array}$ & $\begin{array}{l}0 \\
0 \\
0\end{array}$ & $\begin{array}{l}0 \\
0 \\
0\end{array}$ & $\begin{array}{c}0 \\
3 \cdot 3 \\
0\end{array}$ & $\begin{array}{l}0 \\
0 \\
0\end{array}$ & $\begin{array}{l}0 \\
0 \\
0\end{array}$ & $\begin{array}{l}0 \\
0 \\
0\end{array}$ & $\begin{array}{l}0 \\
0 \\
0\end{array}$ & $\begin{array}{c}0 \\
4 \cdot 4 \\
0\end{array}$ \\
\hline 38 & Control & $\begin{array}{l}\text { Juice } \\
\text { Biopsy } \\
\text { Saline wash }\end{array}$ & $\begin{array}{l}0 \\
0 \\
0\end{array}$ & $\begin{array}{l}0 \\
0 \\
0\end{array}$ & $\begin{array}{c}0 \\
6 \cdot 2 \\
0\end{array}$ & $\begin{array}{c}0 \\
4 \cdot 7 \\
2 \cdot 9\end{array}$ & $\begin{array}{l}0 \\
0 \\
0\end{array}$ & $\begin{array}{l}0 \\
0 \\
0\end{array}$ & $\begin{array}{c}0 \\
4 \cdot 7 \\
0\end{array}$ & $\begin{array}{l}0 \\
0 \\
0\end{array}$ & $\begin{array}{c}0 \\
5 \cdot 7 \\
0\end{array}$ & $\begin{array}{l}0 \\
0 \\
0\end{array}$ & $\begin{array}{l}0 \\
0 \\
0\end{array}$ & $\begin{array}{l}0 \\
0 \\
0\end{array}$ & $\begin{array}{l}0 \\
0 \\
0\end{array}$ & $\begin{array}{l}0 \\
0 \\
0\end{array}$ \\
\hline 39 & Control & $\begin{array}{l}\text { Juice } \\
\text { Biopsy } \\
\text { Saline wash }\end{array}$ & $\begin{array}{l}0 \\
0 \\
0\end{array}$ & $\begin{array}{l}0 \\
0 \\
0\end{array}$ & $\begin{array}{c}0 \\
3 \cdot 3 \\
1 \cdot 0\end{array}$ & $\begin{array}{l}0 \\
0 \\
0\end{array}$ & $\begin{array}{l}0 \\
0 \\
0\end{array}$ & $\begin{array}{c}0 \\
3 \cdot 9 \\
1.0\end{array}$ & $\begin{array}{c}0 \\
0 \\
1.0\end{array}$ & $\begin{array}{c}0 \\
3.3 \\
1.6\end{array}$ & $\begin{array}{c}0 \\
4 \cdot 1 \\
0\end{array}$ & $\begin{array}{l}0 \\
0 \\
0\end{array}$ & $\begin{array}{c}0 \\
4 \cdot 2 \\
0\end{array}$ & $\begin{array}{l}0 \\
0 \\
0\end{array}$ & $\begin{array}{l}0 \\
0 \\
0\end{array}$ & $\begin{array}{l}0 \\
0 \\
0\end{array}$ \\
\hline 40 & Sprue & $\begin{array}{l}\text { Juice } \\
\text { Biopsy } \\
\text { Saline wash }\end{array}$ & $\begin{array}{l}0 \\
0 \\
0\end{array}$ & $\begin{array}{l}0 \\
0 \\
0\end{array}$ & $\begin{array}{c}2 \cdot 5 \\
0 \\
0\end{array}$ & $\begin{array}{c}3 \cdot 2 \\
0 \\
0\end{array}$ & $\begin{array}{l}0 \\
0 \\
0\end{array}$ & $\begin{array}{l}0 \\
0 \\
0\end{array}$ & $\begin{array}{l}0 \\
0 \\
0\end{array}$ & $\begin{array}{l}0 \\
0 \\
0\end{array}$ & $\begin{array}{l}0 \\
0 \\
0\end{array}$ & $\begin{array}{l}0 \\
0 \\
0\end{array}$ & $\begin{array}{l}0 \\
0 \\
0\end{array}$ & $\begin{array}{l}0 \\
0 \\
0\end{array}$ & $\begin{array}{l}0 \\
0 \\
0\end{array}$ & $\begin{array}{l}0 \\
0 \\
0\end{array}$ \\
\hline
\end{tabular}

* See footnote to table I 
TABLE VI

Total possible and actual numbers of aerobic and anaerobic isolates from jejunal juice and jejunal-biopsy homogenates

\begin{tabular}{l|cccc}
\hline $\begin{array}{c}\text { Category } \\
\text { of micro- } \\
\text { organisms }\end{array}$ & $\begin{array}{c}\text { Total possible } \\
\text { number of } \\
\text { isolates }\end{array}$ & juice & biopsy & $\begin{array}{c}\text { Number of } \\
\text { isolates from }\end{array}$ \\
\hline $\begin{array}{l}\text { Aerobes } \\
\text { Anaerobes }\end{array}$ & 360 & 95 & 112 & $>0.05$ \\
\hline
\end{tabular}

isolated from one of the specimens but not from the other (table II). In six pairs there were two groups of organisms (table III) and in 11 pairs there were three or more groups of organisms (table IV) which were not present in one of the specimens. In nine pairs (table V) the jejunal juice grew only one organism or was sterile, but the biopsy grew 3-8 different groups of organisms not found in the luminal fluid. In one pair (table V, no. 40) the jejunal juice yielded organisms, but there was no growth from the biopsy or the washings.

The frequencies with which aerobic and anaerobic organisms were isolated from the luminal aspirates and the biopsies were compared. Nine aerobic and seven anaerobic organisms or groups of organisms were looked for in each specimen, making a total of 360 possible aerobic and 280 possible anaerobic isolates. The number of isolates actually obtained is shown in table VI. There was no significant difference in the frequency of isolation of aerobes between the two groups of specimens, but anaerobes were isolated significantly more frequently from biopsy specimens than from the luminal fluid $(\mathrm{p}<0 \cdot 01)$.

\section{DisCUSSION}

Because both the sampling tube and capsule have to traverse the mouth, oesophagus, stomach and duodenum to reach the first loop of the jejunum, there may have been contamination en route. The first few millilitres of luminal fluid aspirated were discarded in an attempt to minimise contamination of the jejunal juice specimen. In the case of the biopsy, the risk of contamination was less with the Rubin's capsule, which was passed with the knife closed, than with the Crosby capsule which has to be passed in the open position. The mucosal fragments were also washed thoroughly in $2 \mathrm{ml}$ of sterile saline in the hope of removing organisms that might have entered the biopsy capsule in its passage to the upper jejunum. The results of culture of the biopsy washings for the 11 paired specimens in which there were the most discordant results are shown (table V); in nine cases the washings yielded no growth, or only a small number of organisms substantially different from those grown from the biopsy. In only two cases (nos 36 and 39) a very similar pattern of flora was grown from the biopsy and washings. These observations suggest that the flora in at least nine of these 11 biopsies was not due to external 
contamination. The findings with the Crosby biopsy capsule and the Rubin capsule were similar, and this is a further indication that the results were not due to external contamination.

In 13 out of 40 pairs $(32 \%)$ the flora isolated from the jejunal juice and the paired mucosal biopsy was identical or differed by only one organism (tables I and II). Moreover, the total numbers of each organism when expressed per $\mathrm{ml}$ of fluid or per gram of tissue were remarkably similar for each of the 13 pairs. In these 13 subjects, sampling the luminal fluid gave a reasonably representative picture of the total bacterial population at that point in the intestine. At the other extreme, in 10 of the $40(25 \%)$ there was no agreement between the paired specimens (table V). In nine of these, organisms isolated from the jejunal biopsy were not found in the jejunal fluid, which in all except three cases yielded no growth; in the tenth example the jejunal biopsy yielded no organisms but the jejunal juice contained two different organisms.

Isolates were obtained more frequently from the jejunal biopsies than from the luminal fluid; however, this was statistically significant only in the case of the anaerobes. The finding suggests that the micro-environment of the mucosal surface is more favourable for the growth or retention of anaerobes than the micro-environment of the lumen.

There are relatively few studies in which bacterial flora in the lumen and that attached to the mucosa have been compared in man. Plaut et al. (1967) cultured 13 paired luminal fluid and jejunal biopsy specimens from different levels of the small intestine in five healthy North American subjects; although full details of all the organisms recovered are not given, these workers concluded that the organisms in the two situations were similar.

Jarumilinta, Miranda and Villarejos (1976) compared the microbial flora of luminal fluid and jejunal mucosa in paired samples obtained from 18 control subjects and 20 adult patients with acute diarrhoea; they studied 10 groups of organisms. Although they concluded that there were no qualitative differences between the flora grown from the lumen and the biopsy, analysis of their data shows that in 15 out of 38 pairs $(39 \%)$ the flora differed by three or more organisms. In the present study we investigated 16 groups of organisms as compared with their 10 and found that half the pairs differed by three or more organisms. The results of these studies are therefore very similar and indicate that there may be considerable differences between the flora of the lumen and the mucosa (see also Savage, 1970).

In the present study from India only one biopsy, from a patient with tropical sprue, out of 40 was sterile, whereas all the other biopsies, even from the control subjects, had $10^{4}$ organisms or more per gram of tissue. Similarly in the study of Jarumilinta et al. (1976) from Costa Rica, none of the biopsies was sterile and all except two from control subjects had $10^{4}$ organisms or more per gram of tissue. This finding of almost universal contamination or colonisation of the mucosa in these two studies of individuals living in developing countries is quite different from the results of Plaut et al. (1967) who studied North American subjects and found that five out of 15 biopsies were sterile. In studies with germ-free animals (Gordon and Bruckner-Kardoss, 1961; 
Abrams, Bauer and Spring, 1963) it has been shown that the introduction of bacteria has a dramatic effect on the morphology of the small intestine. It is tempting to speculate that the presence of considerable numbers of bacteria in the micro-environment of the epithelial cells may play an important role in the production of the common morphological abnormalities of the jejunal mucosa of apparently healthy subjects in many developing countries (Baker, 1973).

Previous studies from this laboratory failed to show any significant differences between the luminal flora in control subjects and patients with tropical sprue (Bhat et al., 1972). The present study confirms these findings and extends them to include the flora adherent to the jejunal mucosa.

It may be concluded from this study that there are at least two populations of bacteria in the small intestine-one in the luminal fluid and one in the micro-environment of the mucosal epithelial cells. For adequate bacteriological study it is desirable that both these populations be investigated.

\section{SUMMARY}

The aerobic and anaerobic bacterial flora grown from 40 human jejunal aspirates were compared with the flora grown from an intestinal mucosal biopsy obtained simultaneously from the same level. In four paired samples the flora was identical; in nine the flora differed by only one organism. In six pairs the flora differed by two organisms, and in 11 pairs by three or more organisms. In nine pairs the jejunal aspirate grew only one organism or was sterile, but the biopsy showed considerable numbers of organisms. In one pair the jejunal aspirate grew organisms, but there was no growth from the biopsy. It is apparent that for adequate bacteriological study of the intestine, the flora at both sites should be investigated.

\section{REFERENCES}

Abrams, G. D., BaUER, H. AND SPRINZ, H. 1963. Influence of the normal flora on mucosal morphology and cellular renewal in the ileum. A comparison of germ-free and conventional mice. Lab. Invest., 12, 355.

BAKER, S.J. 1973. Geographical variations in the morphology of the small intestinal mucosa in apparently healthy individuals. Pathologia Microbiol., 39, 222.

Bhat, P., Shanthakumari, S., Rajan, D., Mathan, V. I., Kapadia, C. R., Swarnabai, C. and BAKER, S. J. 1972. Bacterial flora of the gastrointestinal tract in southern Indian control subjects and patients with tropical sprue. Gastroenterology, 62, 11.

Cruickshank, R., Duguid, J. P. And Swain, R. H. A. 1965. Medical microbiology, 11 th ed. Livingstone: Edinburgh.

Dubos, R., Schaedler, R. W., Costello, R. and Hoet, P. 1965. Indigenous, normal, and autochthonous flora of the gastrointestinal tract. J. exp. Med., 122, 67.

GORDON, H. A. AND BRUCKNER-KARDOSS, E. 1961. Effects of normal microbial flora on various tissue elements of the small intestine. Acta anat., Basel. 44, 210.

Jarumilinta, R., Miranda, M. AND Villarejos, V. M. 1976. A bacteriological study of the intestinal mucosa and luminal fluid of adults with acute diarrhoea. Ann. trop. Med. Parisit., 70, 165.

Plaut, A. G., Gorbach, S. L., Nahas, N., Weinstein, L., Spanknebel, G. and Levitan, R. 
1967. Studies of intestinal microflora. III. The microbial flora of human small intestinal mucosa and fluids. Gastroenterology, 53, 868 .

RAO, B. R. H. AND RAO, P. S. S. 1958. General health and nutrition survey of the rural population in Pennathur. II. A qualitative study on family meals. Ind. J. med. Sci., 12, 718.

SAVAGE, D. C. 1970. Associations of indigenous micro-organisms with gastrointestinal mucosal epithelia. Am. J. clin. Nutr., 23, 1495. 